\title{
Concise Commentary: Ironing Out the Differences-Healthcare Disparities in the Evaluation of IDA and Diarrhea
}

\author{
Sarah Lopatin ${ }^{1} \cdot$ Sophie Balzora ${ }^{1}$
}

Published online: 7 September 2020

(c) Springer Science+Business Media, LLC, part of Springer Nature 2020

By limiting access to guideline-based high-quality healthcare, racial, and income disparities often negatively impact health outcomes. The foundation for such disparities is multifactorial, oftentimes rooted in systemic causes such as social determinants of health (SDOH).

In this issue of Digestive Diseases and Sciences, AnyaneYeboa and colleagues conducted a single-center retrospective review of patients with concomitant iron deficiency anemia (IDA) and chronic diarrhea in order to determine whether race and insurance status influenced the likelihood of a patient undergoing a guideline-based evaluation including ileocolonoscopy and celiac disease serologies and/or upper endoscopy for the diagnoses of inflammatory bowel disease (IBD) and celiac disease (CD). They found that Black race and public insurance were significantly associated with a decreased likelihood of undergoing an appropriate workup (91\% and 90\% decreased odds, respectively) [1].

Disparities in the diagnosis and management of IBD and $\mathrm{CD}$, two diseases predominantly prevalent in white patients, have gained attention. In 2010, Nguyen et al. demonstrated that Black patients with established IBD were less likely to be under a gastroenterologist's care and more likely to have emergency department visits [2]. In the postpartum IBD population, results from the PIANO Registry showed that race and income level significantly impacted rates of cervical cancer screening and vaccination [3]. Similarly, Lebwohl provided commentary about the underdiagnosis of the non-white "silent minority" of CD patients that is in part secondary to the flawed assumption that CD only affects patients of northern European ancestry [4]. Anyane-Yeboa

Sophie Balzora

Sophie.Balzora@nyumc.org

Sarah Lopatin

Sarah.Lopitan@nyumc.org

1 Division of Gastroenterology and Hepatology, NYU Grossman School of Medicine, 550 First Avenue, New York, NY 10016, USA et al. importantly highlight similar disparities at a crucial stage in patient care, wherein patients are evaluated but fail to receive appropriate diagnostic evaluation despite clear guidelines. Study findings may reflect providers' unconscious biases, exposing the disconnect between the erroneous belief that such diseases only very rarely afflict Black patients and the observed epidemiologic shifts in recent decades showing IBD and CD are increasingly prevalent in nonwhite populations [4, 5]. Failure to perform appropriate diagnostic evaluation and resultant delayed diagnoses can carry significant morbidity, ranging from poor quality of life to the complications of uncontrolled disease.

This study underscores the need to understand the changing landscape of IBD and CD patient demographics, grow cognizant of how SDOH and biases impact provider decision-making, and take deliberate action to create a healthcare workforce that mirrors the diverse backgrounds of our patients in order to combat gastrointestinal-related disparities that result in suboptimal healthcare outcomes for medically underserved groups.

\section{References}

1. Anyane-Yeboa A, Li B, Traboulsi C et al. Black race and public insurance are predictive of inappropriate evaluation of iron deficiency anemia and diarrhea. Dig Dis Sci. (Epub ahead of print). https://doi.org/10.1007/s10620-020-06434-9.

2. Nguyen GC, LaVeist TA, Harris ML, Wang MH, Datta LW, Brant SR. Racial disparities in utilization of specialist care and medications in inflammatory bowel disease. Am J Gastroenterol. . 2010;105:2202-2208.

3. Mao EJ, Sheibani S, Martin C, et al. Preventive health care among postpartum women with inflammatory bowel disease: results from the PIANO registry. Inflamm Bowel Dis. 2019;25:797-802.

4. Lebwohl B. Celiac disease and the forgotten 10\%: the "Silent Minority". Dig Dis Sci. 2015;60:1517-1518. https://doi. org/10.1007/s10620-015-3572-5

5. Aniwan S, Harmsen WS, Tremaine WJ, Loftus EV Jr. Incidence of inflammatory bowel disease by race and ethnicity in 
a population-based inception cohort from 1970 through 2010. Therap Adv Gastroenterol.. 2019;12:1-8.
Publisher's Note Springer Nature remains neutral with regard to jurisdictional claims in published maps and institutional affiliations. 